\title{
Comparison and Analysis between Distinguish Clustering Protocols of Wireless Sensor Networks (A Survey)
}

\author{
Seema Rani Gadai \\ Department of Computer \\ Science \& Engineering \\ BRCM CET, Bahal, MDU, India
}

\author{
Sudesh Kumar \\ Department of Computer \\ Science \& Engineering \\ BRCM CET, Bahal, MDU, India
}

\begin{abstract}
A WSN as a finest having rules i.e. energy (power) validation from every sensor hub is comprised the battery or energy device with a restricted energy (power) provide to job. Likewise, not easy task to replace the battery in different environment there may be very rough or exhaustive in a few different situations. On the other side, the wireless sensor network says that there may be enough energy to complete the task or can gather the information easily. There are so many clustering protocol to maximize the life cycle of network in WSN. This will be a typical to task to create the mind relax system that can help to collect the data and as well as less energy usage. In this paper, focus on different clustering protocols with different parameters that suggest which one protocol is better for the nodes into hostile area \& further to improvement of energy usage should be less.
\end{abstract}

\section{Keywords}

Sensor network, energy efficiency, clusters head.

\section{INTRODUCTION}

A Wireless Sensor Network (WSN) is an engaged remote system that includes various sensor hubs orchestrate in a predetermined territory for checking environment conditions, for example, temperature, pneumatic force, mugginess, light, movement or vibration, etc. The sensor hubs are normally customized to gather data from encompassing environment and transfer to the base station for remote client access through distinguish correspondence advances. Figure 1 shows general wireless sensor system structural engineering. Ordinarily, a sensor hub is a little gadget or bit that comprises of four fundamentals are as follows:

1. Processing subsystem for information preparing and information putting away.

2. Sensing subsystem for information gathering from its surroundings environments.

3. Energy supply subsystem which is a force hotspot for the sensor node.

4. Wireless correspondence subsystem for information transmission.

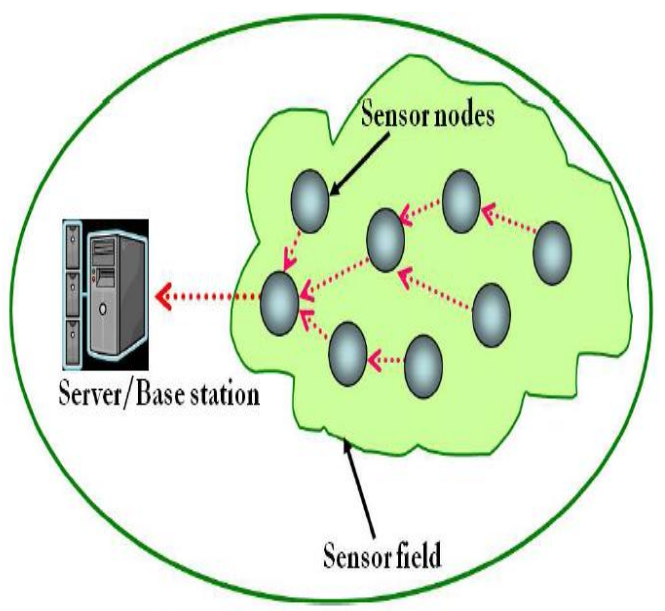

Fig 1 WSN architecture

Be that as it may, sensor hubs have little memory, moderate handling speed, and restricted vitality supply. These confinements are run of the mill attributes of sensor hubs in wireless sensor networks.

Sensor networks are needed in the applications like environment monitoring, industrial control units, military applications and in the context aware computing environments. Based on this critical expectation, in many crucial WSN applications the sensor nodes are often deployed randomly in the area of interest by relatively uncontrolled means (i.e., dropped by a helicopter) and they form a network in an ad hoc manner $[1,2]$.

A wireless sensor networks as a rule has vitality limitation because of every sensor hub needs battery with a restricted vitality supply to work. Likewise, reviving or supplanting sensor battery may be less than ideal and unfeasible in a few situations. On the other side, the Wireless sensor system ought to work sufficiently long to consideration fulfill the application prerequisites. Along these lines, vitality protection is a fundamental matter in the arrangement of Wireless sensor systems. There are disparate ways to deal with protect vitality tradition and drag out the system lifetime or prolong in WSN. The key way to deal with improve vitality use in WSN is the development of vitality mindful system conventions. In this dissertation display an audit of directing and bunching calculations for force protection in Wireless sensor systems. This additionally show a force mindful bunching strategy for improving the system lifetime and also growing the quantity of effectively conveyed bundles and diminishing the system delay time. 


\section{ENERGY DISSIPATION AND ENERGY WASTE IN WSN}

Ideally, this would like the wireless sensor network to perform its functionality as long as possible. Optimal routing is based on energy constrained networks is not practically feasible (because it requires future knowledge). However, this can soften our requirements towards a statistically optimal technique, which increase the network functionality considered over all possible future activity. A technique is energy efficient (in contrast to 'energy optimal') when it is statistically optimal and causal. Since energy efficiency is more important for wireless sensor networks than any other networks, more research works have already been done in routing in WSN. In general, data transmission in wireless communication takes more energy than data processing. Whenever the nodes are transmitting more data proportionately their battery power also get reduced. To reduce the data size can go for data fusion or data aggregation techniques. Data fusion is that in which the sensed data from different nodes are fused at certain point suitable for the transmission in its reduced data size. And, data aggregation can be performed at an intermediate node to eliminate data redundancy, which can reduce the total amount of traffic in the network and thus improve the energy efficiency of the network.

\section{ENERGY EFFICIENCY IN ROUTING}

Ideally, we would like the wireless sensor network to perform its functionality as long as possible. Optimal routing is based on energy constrained networks is not practically feasible (because it requires future knowledge). However, we can soften our requirements towards a statistically optimal technique, which increase the network functionality considered over all possible future activity. A technique is energy efficient (in contrast to 'energy optimal') when it is statistically optimal and causal. Since energy efficiency is more important for wireless sensor networks than any other networks, more research works have already been done in routing in WSN. In general, data transmission in wireless communication takes more energy than data processing. Whenever the nodes are transmitting more data proportionately their battery power also get reduced. To reduce the data size we can go for data fusion or data aggregation techniques. Data fusion is that in which the sensed data from different nodes are fused at certain point suitable for the transmission in its reduced data size. And, data aggregation can be performed at an intermediate node to eliminate data redundancy, which can reduce the total amount of traffic in the network and thus improve the energy efficiency of the network.

Data aggregation is a technique for removing data redundancy and improving energy efficiency in WSNs. The basic idea is to combine the data received from different sources (sensor nodes) so that the redundancy in the data is minimized and the energy consumption for transmitting the data is reduced in the aggregation task. There are different techniques for data aggregation in WSNs. Since conserving energy is one of the most important challenges in data aggregation, an efficient data aggregation technique should be able to balance the amount of energy consumed by each sensor node in each round of data gathering in WSNs.

Study on energy efficient routing in WSN are follows.

\section{A. Leach:}

To overcome the shortcomings of conventional routing and data dissemination protocols, which run on top of no layered or flat network architectures, a clustering - based protocol, called low energy adaptive clustering hierarchy (LEACH) was proposed in Ref. [3]. LEACH Shown in Fig 2 is based on a data aggregation or data fusion technique that combines or aggregates the original data into a smaller size of data that hold only meaningful information of all individual sensors. For this purpose, LEACH divides a network into many clusters of sensor nodes, which are constructed by using localized coordination and maintain not only to reduce the amount of data that are transmitted to the sink or base station, but also to make routing and data dissemination more reliable, scalable and robust. Given that the energy dissipation of the sensors depends on the distance and the data size to be transmitted, LEACH attempts to transmit data over short distances and reduce the number of transmission and reception operations.

Step1: The eligible cluster head nodes will be issuing a notification to the nodes coming under its range to become a cluster member in its cluster. The node will be accepting the offer based upon the Received Signal Strength (RSS).

Step2: In this step the nodes will be responding to their selected cluster heads. After receiving response from the nodes the cluster head have to make a TDMA scheme and send back to its cluster members to intimate them when they have to pass their information to it.

Step3: The data collected by the each sensor node will be given to the cluster head $(\mathrm{CH})$ during its time period and on all other time the cluster members radio will be off to reduce it energy consumption of sensor node. Here in the LEACH protocol multi cluster interference problem was resolved by using unique CDMA (Code Division Multiple Access) codes for each cluster. It supports to prevent energy drain for the same sensor nodes which has been

selected as the cluster head, using randomization for each time cluster head would be changed. The cluster head of each cluster is responsible for collecting data from its cluster members and fuse \& aggregate it also. Finally each cluster head will be forwarding the fused data to the base station (BS). When compared with its previous protocols LEACH has shown a considerable improvement in case of energy consumption. 


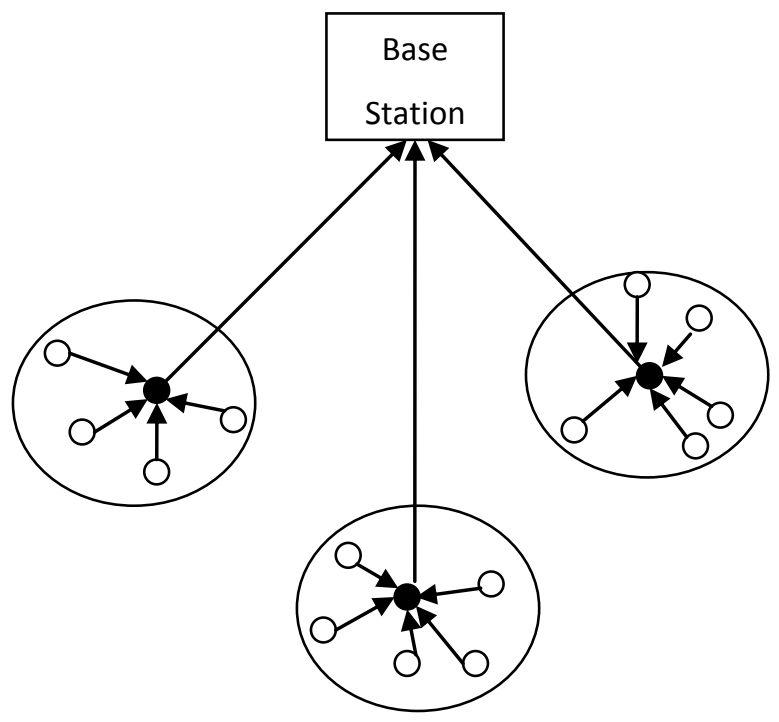

Figure 2: LEACH

\section{B. Heed}

The Hybrid Energy Efficient Distributed clustering protocol (HEED) is also a cluster heads selection base protocol focused on a hybrid of the nodes residual energy and some communication costs.

- Same LEACH, the clustering process arrangement is completely distributed and terminates in a fixed number of rounds. But in contrast to LEACH \& HEED guarantees good cluster head sharing and assumes that cluster heads have relatively high average residual energy compared to regular nodes.

- The drawback of HEED is that it is based on the supposition that nodes can tune their communication range through transmission power: low energy levels are used for intra cluster communication, higher energy levels for inter cluster communication.

- Real RF phenomena such as external intrusion and multipath fading make it hard to forecast the communication range from the transmission energy, especially indoors. This causes HEED to be of tiny practical use in a real employment.

Hybrid energy efficient and distributed (HEED) clustering technique for ad hoc sensor networks [4]. This was proposed with four key goals:

1. Enhancing network lifetime by distributing energy spending,

2. Terminating the clustering task within a constant number of rounds

3. Reducing control overhead

4. Producing fine distributed cluster heads and solid clusters.

HEED occasionally elects cluster heads based on a hybrid of two clustering parameters:

- The primary parameter is the residual energy (RE) of each sensor node
- Secondary parameter is the intra cluster communication cost as a function of neighbor proximity or cluster density

The primary parameter is used to probabilistically elect an early set of cluster heads while the secondary parameter is used for breaking ties. The clustering task at each sensor node needs multiple rounds. Every round is lengthy to get messages from any neighbor within the cluster range. As in LEACH, a preliminary percentage of cluster heads in the network, C prob, is predefined. The parameter $\mathrm{C}$ prob is only used to bind the preliminary cluster head announcements and has no direct impact on the final cluster structure. In HEED, each sensor node sets the probability

$\mathrm{CH}$ prob of becoming a cluster head as follows

\section{Pegasis}

$$
\mathrm{CH}_{\text {prob }}=\mathrm{C}_{\text {prob }} \frac{E_{\text {residua }}}{E_{\text {max }}}
$$

Instead of creating any cluster, Power efficient Gathering in Sensor Information Systems (PEGASIS) in Fig. 3 [5] creates a chain of sensor nodes where each sensor node transmits and receives data from a neighbor and at a time only one node can send data to the sink or BS. Data are aggregated at the nodes when they move from one node to another node. It is shown that PEGASIS can outperform LEACH by about 100 300\% for distinguish network sizes and network topologies. But, it causes long latency for nodes located at a distance on the chain from BS. It is also subject to single point of failure in case the single leader on the chain fails which is also the reason for performance bottleneck of the system.

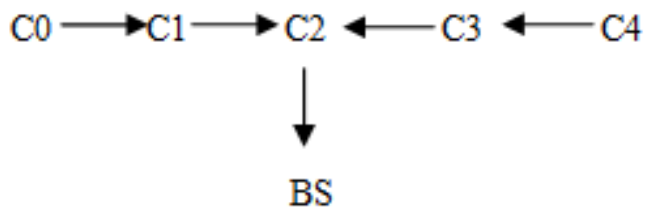

Figure 3: Chaining in PEGASIS

Though the Cluster based protocols like LEACH have shown a factor of 8 improvements when compared with its previous protocols further improvements were done by forwarding the packets to only one neighbor of the node. This technique had been named as, PEGASIS [5]. Instead of forwarding the packets from many cluster heads as like in LEACH protocol here in PEGASIS each node will create a chain structure to the base station through which the data would be forwarded to the BS node. Here in PEGASIS energy efficient is gain by transmitting the data to only one of its neighbor node. There the collected data is fused and the fused data will be forwarded to its immediate one hop neighbor. Since all the nodes are doing the data fusion at its place there is no rapid depletion of energy for the nodes present near the Base station. Also in this technique each node will be getting the chance to forward the gathered data to the base station. But when the sensor measurements are aggregated to be a single packet, only fraction of the data generated by the sensor is given to the base station. In other applications when the individual sensor measurement is needed it fails to give it to base station. But apart from the method of the routing protocol can make the sensor network database to follow the multi resolution scheme where the aggregated data will be present in the root node and the finer data can be obtained by further tree traversal approach. 


\section{Teen}

Threshold Sensitive Energy Efficient Network Protocol (TEEN) [6] combines both data-centric and hierarchical approach. Instead of having a flat clustering of nodes like LEACH or PEGASIS, TEEN forms multi-level hierarchy of nodes by grouping closer nodes into clusters on the first level, then on the second level and so on until the sink node is reached. Figure 3, which is redrawn from [6], shows this method of clustering. TEEN tries to reduce energy consumption by reducing the number of transmissions with the aid of two thresholds as broadcast by the cluster heads after the clusters are created - hard and soft thresholds. Hard threshold refers to the minimum possible attribute value that causes a sensor node to switch on its transmitter and transmit the sensed data to the cluster head, whereas a node sensing an attribute value at or beyond the hard threshold will transmit the sensed data only when the attribute value changes by an amount equal to or greater than the soft threshold. Although TEEN is quite energy-efficient, it is not useful for applications that require periodic reporting of the sensed data. Most importantly, the forming of multi-level hierarchy, implementing different thresholds and attribute-based naming impose huge overhead and complexity to this approach.

\section{E. Apteen}

An advancements to TEEN was proposed in [7] called Adaptive Threshold Sensitive Energy Efficient Network Protocol (APTEEN) which removes the limitation of TEEN by incorporating the periodic reporting of sensed data along with the reactive response. Figure 4 shows the architecture of APTEEN which remains the same as TEEN as a result of which APTEEN inherits the drawbacks of TEEN as well.

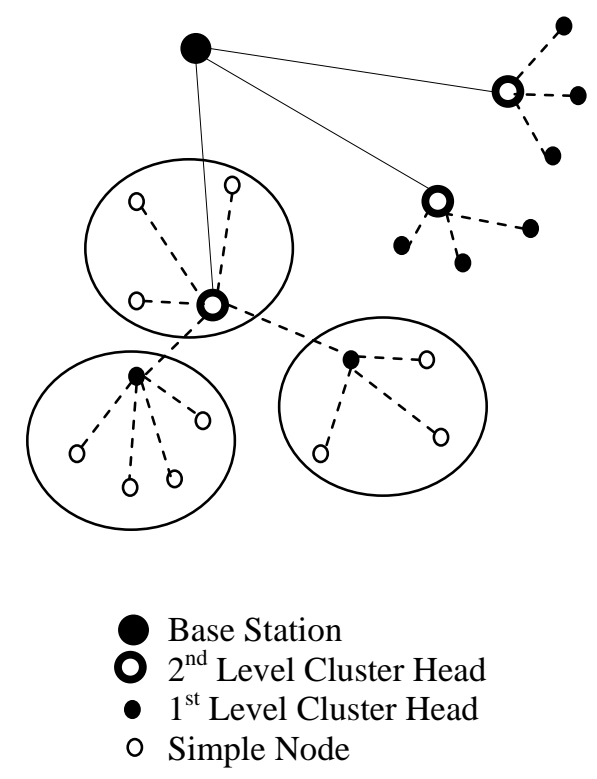

Fig. 4 Hierarchical Clustering in TEEN and APTEEN

\section{F. Dwehc}

Distributed weight - based energy - efficient hierarchical clustering (DWEHC) is another clustering algorithm proposed to achieve balanced cluster sizes and optimize intracluster topologies for WSNs [8][12]. Both DWEHC and HEED share some similarities including no assumptions about network size and density, and considering residual energy in the process of cluster head $(\mathrm{CH})$ selection, but assumes that sensor nodes are location aware and transmit at the same fixed power levels. The cluster radius (R); that is, the farthest transmission distance from one cluster member node to its $\mathrm{CH}$ is fixed for the whole network. After individually running seven iterations on each node, DWEHC generates a multi hop intra-cluster structure in which a cluster head is at the root and member nodes are in a breadth - first order. Figure 5 illustrates an intra-cluster example, in which each cluster has a hierarchical structure with one $\mathrm{CH}$ and the child nodes of the first level, second level, and so on. Each cluster has multi levels of child nodes. Since there are no assumptions about the size and topology of the network, the number of levels within one cluster is determined by the cluster radius and the minimum energy path from one member node to its cluster head is established by DWEHC. Each node only responds to its nearest parent' $s$ request, and that parent then responds to its own parent until the data reaches the cluster head. Time division multiple access (TDMA) is used for intra-cluster communication and the cluster heads contend for the channel using the 802.11 protocol to send data to the base station.

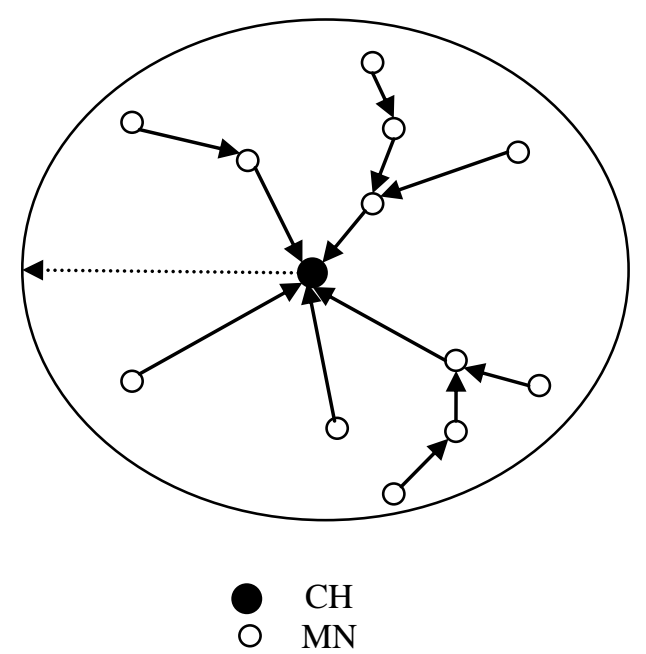

Fig. 5 Structure of Multi-level Cluster in DWEHC

Different from LEACH and HEED, DWEHC creates a multilevel structure for intra-cluster communication and limits a parent node's number of children. Moreover, the only locally calculated parameter weight is defined for $\mathrm{CH}$ election in DWEHC. After locating the neighboring nodes in its area, each node calculates its weight according to:

$\mathrm{W}_{\text {weight }}(s)=\frac{E_{\text {residual }}(s)}{E_{\text {initial }}(s)} \times \sum_{u} \frac{R-d}{6 R}$

Where $E_{\text {residual(s) }}$ and $E_{\text {initial(s) }}$ are respectively residual and initial energy at node $\mathbf{s}, \mathbf{R}$ is the cluster range that corresponds to how far from the $\mathrm{CH}$ to a node inside a cluster and $\mathbf{d}$ is the distance between node $\mathbf{s}$ and the neighboring node $\mathbf{u}$. In a neighborhood, according to Equation (1), the node with largest weight would be elected a $\mathrm{CH}$ and the other nodes become members. At this stage, MNs are considered as 1level nodes and communicate directly with the $\mathrm{CH}$. A MN can progressively adjust such membership in order to reach a $\mathrm{CH}$ using the least amount of energy. Given the node's knowledge of the distance to its neighbors, it can assess whether it is better to stay a 1-level member or become a h-level one where $\mathrm{h}$ is the number of hops from the $\mathrm{CH}$ to itself. If a $\mathrm{MN}$ can save energy while reaching its $\mathrm{CH}$ with more than one hop, it will become an h-level member. The process continues until all nodes achieve the most energy-efficient intra-cluster 
topology. Energy consumption for communicate in a cluster can be computed according to node's knowledge of the distance to its neighbors. To limit the number of levels, every cluster is assigned a cluster range within which MNs should lay. The structure of multi-level cluster in DWEHC is illustrated in Figure 5. After running DWEHC, a node either becomes a $\mathrm{CH}$ or becomes a child in a cluster, and a node is covered by only one $\mathrm{CH}$.

Intra-cluster communication is performed by TDMA. Each parent node polls its direct children and forwards the data to its parent node until the data reaches the $\mathrm{CH}$. The parent node may aggregates several data packets from its children together with its own data into one packet. For inter-cluster communication, the $\mathrm{CHs}$ poll their first-level children, including their own data, and transmit to the BS.

The following is the advantages of DWEHC: (1) Like HEED, it is a fully distributed clustering method that is based on a function of the sensor's energy reserve and the proximity to the neighbors for $\mathrm{CH}$ election; (2) Considering energy reserves in $\mathrm{CH}$ election, DWEHC generates more wellbalanced $\mathrm{CHs}$ distribution and achieves significantly lower energy consumption in intra-cluster and inter-cluster routing than HEED; (3) The clustering process of DWEHC terminates in a few iterations, and does not depend on network topology or size.

Some disadvantages of DWEHC are summarized as follows:

(1) Similar to LEACH, single-hop inter-communication, directly from $\mathrm{CHs}$ to the BS, is performed in DWEHC. Thus
DWEHC may result in significant amount of energy consumption, and is not applicable to large-region networks; (2) In the process of cluster formation; the iterative nature in both DWEHC and HEED produces a relatively high control message overhead compared to other protocols.

\section{COMPARISON OF DIFFERENT CLUSTERING ROUTING PROTOCOLS IN WSNS}

In this section, comparison of different energy efficient clustering routing algorithms for WSNs. Table formation summarize the categories and differences of the clustering routing protocols in WSNs according to a variety of clustering attributes as shown in Table I.

\section{CONCLUSION}

WSN, by nature, is extremely energy constrained thereby forcing the routing protocol designers to go for energy efficient design. In this paper routing protocols showed the main approaches to energy saving methods in Wireless Sensor Network, and a comprehensive list of the EER protocols for WSN has been studied. These energy saving methods are basically used to increase the life time of sensor nodes in wireless sensor networks. So this paper emphasize on developing convenient techniques to reduce the energy consumption of the sensors by this approach.

Table 1. Classification of Different Clustering Routing Protocols in WSNs

\begin{tabular}{|c|c|c|c|c|c|c|}
\hline Parameters & LEACH & HEED & PEGASIS & TEEN & APTEEN & DWEHC \\
\hline Intra-cluster routing & single-hop & single-hop & simple-hop & single-hop & simple-hop & multiple-hop \\
\hline Inter-cluster routing & single-hop & $\begin{array}{c}\text { single-hop } \\
\text { multiple-hop }\end{array}$ & single-hop & multiple-hop & multiple-hop & single-hop \\
\hline Class & Hierarchical & Hierarchical & Hierarchical & Hierarchical & Hierarchical & Hierarchical \\
\hline Scalability & Very Low & Moderate & Very Low & Low & Low & Moderate \\
\hline Energy Efficiency & High & High & High & Very High & Moderate & Very high \\
\hline $\begin{array}{l}\text { Variability of cluster } \\
\text { count }\end{array}$ & variable & variable & variable & fixed & variable & variable \\
\hline Data Aggregation & Yes & Yes & Yes & Yes & Yes & Yes \\
\hline $\begin{array}{l}\text { Difference of } \\
\text { capabilities }\end{array}$ & homogeneous & homogeneous & N/A & homogeneous & homogeneous & homogeneous \\
\hline Hop Communications & Single-hop & Single-hop & Multi-hop & Multi-hop & Multi-hop & Single-hop \\
\hline Control manners & distributed & distributed & distributed & distributed & distributed & distributed \\
\hline Load Balancing & Moderate & Moderate & Moderate & Good & Moderate & Very Good \\
\hline
\end{tabular}

\section{REFERENCES}

[1] K. Sohrabi et al. (2000), Protocols for self-organization of a wireless sensor network, IEEE Personal Communications, 7(5).

[2] R. Min et al. (January 2001), Low power wireless sensor networks, in Proceedings of International Conference on VLSI Design, Bangalore, India.

[3] W.B. Heinzelman, A.P. Chandrakasan, H.Balakrishnan (2002), "Application specific protocol architecture for wireless micro sensor networks", IEEE Transactions on Wireless Networking.

[4] O. Younis and S. Fahmy (2004), HEED: A hybrid, energy-efficient, distributed clustering approach for Ad
Hoc sensor networks, IEEE Transactions on Mobile Computing, 3(4), 366-379.

[5] S. Lindsey, C. Raghavendra (2002), "PEGASIS: PowerEfficient Gathering in Sensor Information Systems," IEEE Aerospace Conference Proceedings, Vol. 3. No. 916.

[6] A. Manjeshwar and D.P. Agrawal (April 2001), “TEEN: a protocol for enhanced efficiency in wireless sensor networks", in Proceedings of the 1st International Workshop on Parallel and Distributed Computing Issues in Wireless Networks and Mobile Computing, San Francisco, CA.

[7] A. Manjeshwar and D.P. Agrawal (April 2002), "APTEEN: a hybrid protocol for efficient routing and 
comprehensive information retrieval in wireless sensor networks", in Proceedings of the 2nd International Workshop on Parallel and Distributed Computing Issues in Wireless Networks and Mobile computing, Ft. Lauderdale, FL.

[8] Ding, P.; Holliday, J.; Celik (June 2005), A. Distributed Energy Efficient Hierarchical Clustering for Wireless Sensor Networks. In Proceedings of the 8th IEEE International Conference on Distributed Computing in Sensor Systems (DCOSS), Marina Del Rey, CA, USA.

[9] Congfeng Jiang, Daomin Yuan, Yinghui Zhao (2009) "Towards Clustering Algorithms in Wireless Sensor Networks-A Survey", IEEE Communications Society publication in the WCNC 2009 proceedings.

[10] Soroush Naeimi, Hamidreza Ghafghazi, Chee-Onn Chow, Hiroshi Ishii (May 2012) "A Survey on the
Taxonomy of Cluster-Based Routing Protocols for Homogeneous Wireless Sensor Networks", Sensors (Basel). 2012; 12(6): 7350-7409, PMC ID: PMC3435979.

[11] Naveen Sharma, Anand Nayyar (January 2014) "A Comprehensive Review of Cluster Based Energy Efficient Routing Protocols for Wireless Sensor Networks", International Journal of Application or Innovation in Engineering \& Management, Volume 3, Issue 1.

[12] Sanjeev Kumar Gupta, Neeraj Jain, Poonam Sinha January 2013, "Clustering Protocols in Wireless Sensor Network s : A Survey", nternational Journal of Applied Information Systems (IJAIS) -ISSN: 22490868Foundation of Computer Science FCS, New York, USAVolume 5- No.2, www.ijais.org. 